\title{
Design, Development and Characterization of Graphene Sand Nano-Composite for Water Filtration
}

\author{
Shahid Hussain Abro*a, Alidad Chandio ${ }^{b}$ Abdulaziz S. Alaboodi ${ }^{b}$ and, Iftikhar A.Channa \\ ${ }^{a}$ Department of Materials Engineering, NED University of Engineering and Technology, Karachi, Pakistan. \\ ${ }^{b}$ Department of Metallurgical Engineering, NED University of Engineering and Technology, Karachi, Pakistan \\ ${ }^{c}$ Friedrich University of Erlangen, Nuremberg, Germany \\ ${ }^{\mathrm{d} D e p a r t m e n t ~ o f ~ M e c h a n i c a l ~ E n g i n e e r i n g, ~ Q a s s i m ~ U n i v e r s i t y, ~ S a u d i ~ A r a b i a ~}$
}

(received August 30, 2018; revised December 19, 2019; accepted March 27, 2019)

\begin{abstract}
Water purification and filtration is a global issue and many researchers are engaged to resolve this problem by adopting the scientific approach, graphene sand composite was prepared through biosynthesized technique. River sand was used in this context to remove the impurities already present in the sand $0.1 \mathrm{M}$ nitric acid treated the sand and the product was powder black in colour, referred as GSC, graphene sand composite. SEM, XRD and FTIR characterization was used to analyze the results. SEM images showed nano sized layers or sheets of graphene extending outwards. The XRD peak represents the multi lagered graphene structure which is formed by the treatment of the composite with acid and application of the high temperature during experiment UV-visible spectroscopy results successfully reveals the filtration difference between mud water and filtered water.
\end{abstract}

Keywords: graphene, biosynthesized, sugar anchoring, sand particles, water filtration.

\section{Introduction}

Graphene is an exceptional 2-dimensional allotrope of carbon(Abdel majid et al., 2018; Chong yang et al., 2018; Chandra et al. 2010). The applications of graphene along with graphene based compounds composites for the removal of pollutant, filtration, environmental remediation, electronic circuits, solar cells and several chemical, industrial and medical processes are being explored.Graphene can be prepared by two distinct methods (i) Graphite is chemically transformed to GO, graphite oxide followed by reducing the graphite oxide by $\mathrm{N}_{2} \mathrm{H}_{4}$ (hydrazine) to reduce graphene oxide (ii) anchoring of graphite oxide and reduced graphite oxide upon sand particles either by applying heat treatment or by covalently bonding on silica directly as well as by applying molecular binders. Although many different chemicals can also be used to synthesize of RGO such as $\mathrm{N}_{2} \mathrm{H}_{4}$ (hydrazine), $\left(\mathrm{P}_{2} \mathrm{O}_{5}\right)$ and $\left(\mathrm{K}_{2} \mathrm{~S}_{2} \mathrm{O}_{8}\right)$ as a result it can generate unwanted hazardous products such as, $\left(\mathrm{P}_{2} \mathrm{O}_{3}\right),\left(\mathrm{SO}_{2}\right)$, etc. (Lujanienë et al., 2017; Das et al., 2014). This may require laborious work such as postsynthesis cleaning. (Jisoo et al., 2018). Many graphene based composites have been developed so far even with and without metal oxides for water filtration applications but for success of such materials, cost is always the main criterion and therefore, it becomes compulsory

*Author for correspondence; E-mail: engrabro@neduet.edu.pk that new and advanced prospective techniques should always be used for producing such products (Xiao et al., 2016; Savag and Dillo 2005). Graphene sand composite is the insitu development of graphenic stuff material supported upon the surface of river's sand which requires additional binders. But in research process no harmful residues were left when sugars readily decomposed into carbon. (Dreyer and Sungjin, 2010). Waste water can be treated by bio-synthesized graphene process imposing the anchoring of graphene upon the river's sand and make unlikely to change the graphene as a result it more suitable for treatment of flowing water framework (Dreyer et al., 2012). The method used in present research work for the synthesis of composite is different from conventional composite manufacturing because it is a nano composite. (Poornima Parvathi and Umadevi, 2016; Bykkam et al., 2013; Yang and Chong 2010) after removing from furnace it was treated with sulphuric acid to activate it. (Peng et al., 2015).

It is most desirable to develop and produce the graphene adsorbent in economical way as well as it is eco-friendly and use for water purification and filtration. A scientific approach is made in this work to design and establish the unique technique which is used for advance and traditional method to purify the drinking water. Graphene Sand Composite (GSC) was successfully 
developed and the results were confirmed by advanced characterization techniques. (Song et al., 2018; Chao et al., 2013).

\section{Materials and Methods}

Materials used in the process were almost easily available and purchased from the local market sugar, sulphuric acid, nitric acid, activated charcoal and de-ionized water. The sand was obtained from the sea view, having the particle size of 180 microns. The overall experimental work was performed in the following steps:

Synthesis of GSC. Sand was first washed with $0.1 \mathrm{M}$ nitric acid in order to remove any contaminants present in the sand. These contaminants could be different ions causing any unfavourable reaction during the process. Then sand was dried subsequently in air for one day. This washed sand was used in all the experimental titerations, performed as shown in Fig. 1 (A). The dried sample was put inside of the graphite crucible.

Preparation of sugar sand solution. Molar sugar solution was prepared by adding $342 \mathrm{~g}$ of sugar in 1 Liter of deionized water. This was done by mixing the solution thoroughly. Then $20 \mathrm{~g}$ of sand (particle size 180 micron) was added to the solution.

Mixing and drying on magnetic stirrer. The beaker containing the solution of sugar and sand was placed on the hot plate magnetic stirrer for about $6 \mathrm{~h}$ with

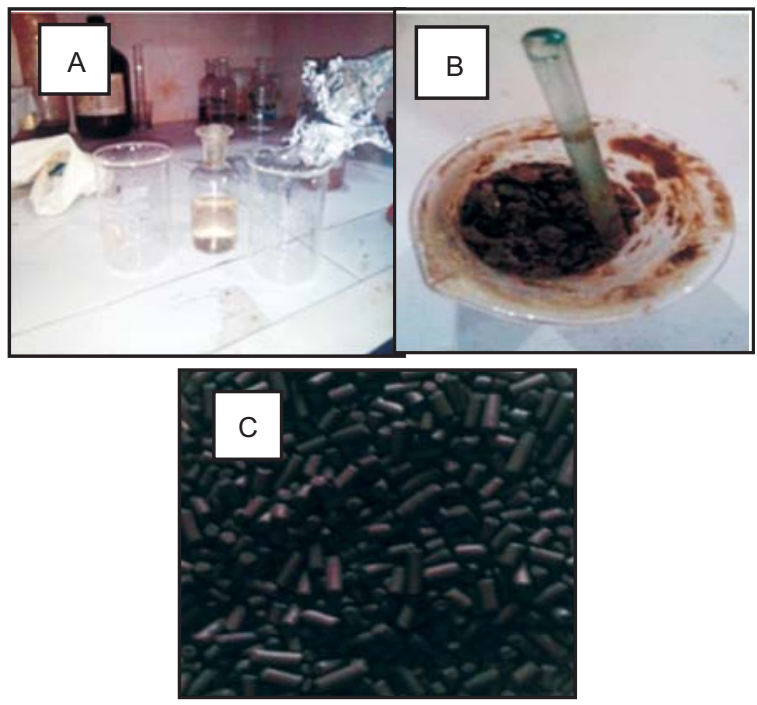

Fig. 1. (A) sand washing. (B) Sample obtained after hot plate stirring. (C) Crucible covered with charcoal. continuous stirring and approximatly $85^{\circ} \mathrm{C}$ to evaporate the water. The sample was grey colour of the solution as shown in Fig 2.1 and 2.2

The crucible was covered by activated charcoal dispersed from all sides to protect for the reducing environment as shown in Fig1 (C). Heat treatment was performed in a muffle furnace. Fig. (2) first it was heated to about $200^{\circ} \mathrm{C}$ for $1 \mathrm{~h}$ then it was held there for about $1 \mathrm{~h}$ to unformly heat all the samples and heated to $750^{\circ} \mathrm{C}$ for $1 \mathrm{~h}$ and held at this temperature for about $3 \mathrm{~h}$ and then furnace wash cooled. After heat treatment, black sample obtained which was graphene sand composite as shown in Fig. 2.1. The sample was treated with sulphuric acid (for every $5 \mathrm{~g}$ of sample $20 \mathrm{ml}$ of acid was used) for about $1 \mathrm{~h}$ to active it and washed with deionized water and filtered by means of filter paper (Theruvak kattil Sree nivasan, 2013). The resulting sample was then dried on the hot plate for about half hour at $120^{\circ} \mathrm{C}$ as shown in Fig. 2.2.

Now, again followed same methodology in this titration. The particle size of sand was reduced from 180 microns to 3 microns through ball milling of the sample for about $75 \mathrm{~h}$. The sample obtained from ball milling
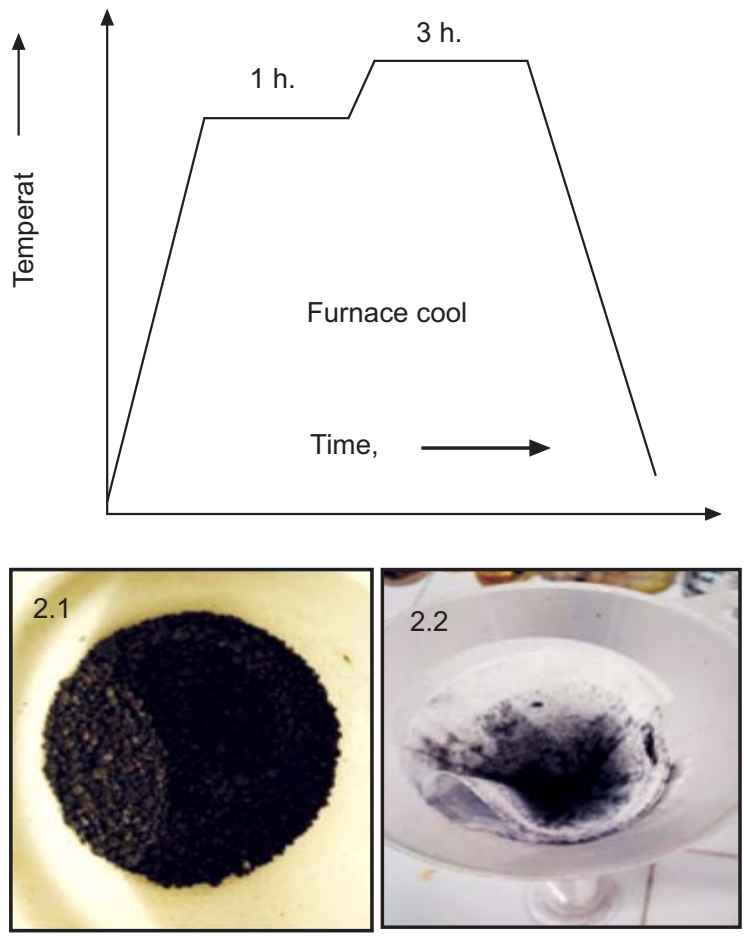

Fig. 2. Heat treatment cycle. Fig. 2.1 GSC powder after heat treatment. Fig. 2.2 Filtering sample 

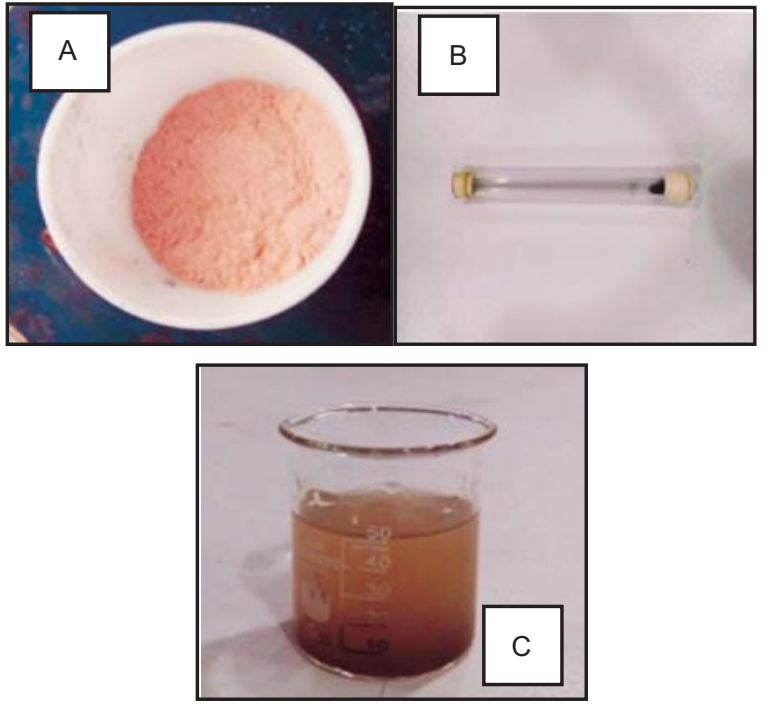

Fig. 3. (A) GSC obtained after treatment. The sample obtained was whitish brown. (B) Filter Design with simple tube covered at one end with cloth. (C) Dirty water combined with mud.

machine was very fine powder of white colour as shown in Fig. 3 (A).

Water filtration is a simple filteration which was made by the glass tube closed at one end and allow only water to pass through the sample and also hold the sample in place as shown in Fig. 3 (B). The water from the mud was collected in a beaker (Fig. $3 \mathrm{C}$ ) and passed through the filter tube and collected in the bottles for further testing of this purified water.

\section{Results and Discussion}

Synthesis and characterization of GSC. X-ray diffraction. The XRD results of the GSC sample in the powdered form (180 micron) is shown in Fig. 4. The peak obtained at $20.93^{\circ}$ represents the multi layered graphene structure which is formed by the treatment of the composite with acid and application of the high temperature in the procedure. The d-spacing for GSC is also higher, than the pristine graphene because of this treatment and equal to $0.402 \mathrm{~nm}$.

The characteristic peaks of graphene were obtained at $26.6^{\circ}, 42.4^{\circ}, 44.7^{\circ}$, and $54.8^{\circ}$. The peak for multi layered graphene was obtained at $20.9^{\circ}$ degrees and the corresponding d-spacing is $0.402 \mathrm{~nm}$. Almost similar results were obtained when the sand size was reduced to 3 microns by ball milling for 60 hours as shown in Fig. 4.

FTIR spectroscopy. The FTIR results as shown in Fig. 5 which verifies the proper and perfect graphitization of 180 micron sand. It clearly indicate the absorption

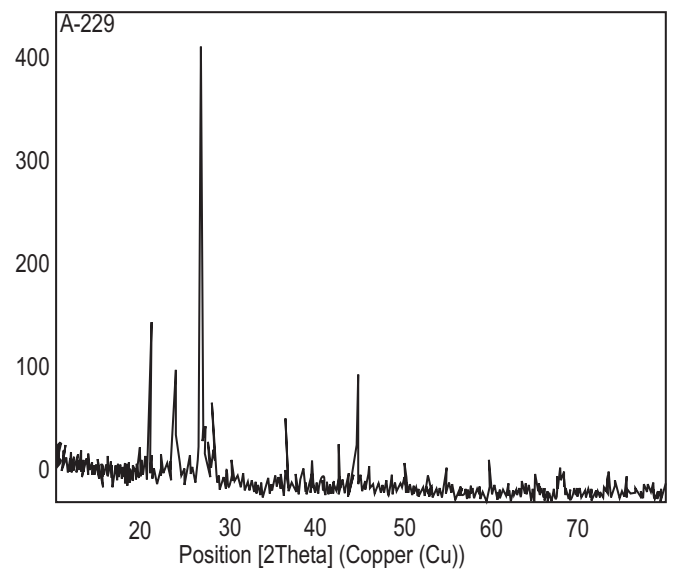

Fig. 4. XRD pattern for 180 micron GSC.
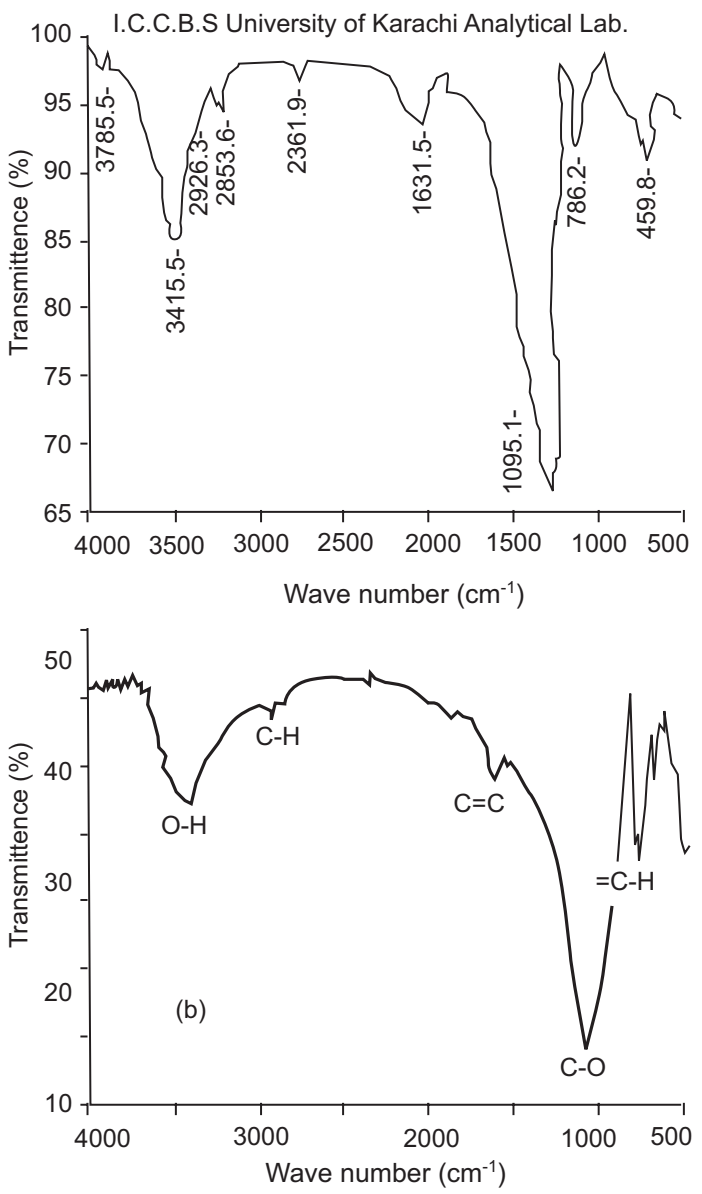

Fig. 5. FTIR of GSC sample. 
band at $1631.5 \mathrm{~cm}$, that was closed similarity straighten at $\mathrm{C}=\mathrm{C}$ and indicated the sketchy graphene structure. An absorption band present at $1095.1 \mathrm{~cm}$ indicates the $\mathrm{C}=\mathrm{O}$ sketchy graphene structure. However, the band straighten at $3415.5 \mathrm{~cm}$ indicates $\mathrm{O}-\mathrm{H}$, which represents the oxygen functionality present at that point. The band at $2925 \mathrm{~cm}$ respresents $\mathrm{C}-\mathrm{H}$ and at 778 is for $\mathrm{C}-\mathrm{H}$.

Results of filtration. $U \boldsymbol{V}$ visible spectroscopy. Two Fig. 6 (A) and (B) describes elementary UV-visible absorption spectrum for mud water and filtered mud water sample. The values on y-axis indicate the amount of light absorbed.

If the Y-axis value is greater than higher the wavelength is absorbed.The large difference was obtained between absorption of mud and filtered mud water.
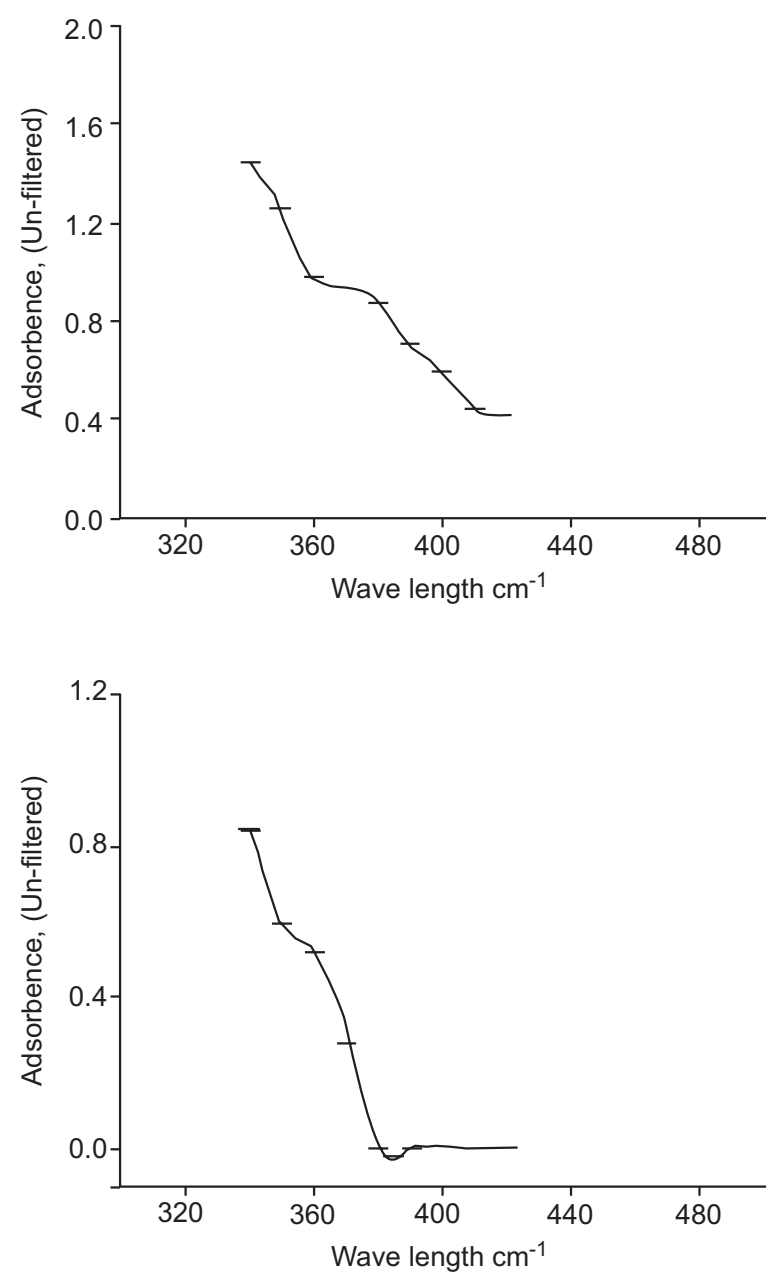

Scanning electron microscopy images at different resolutions showed layers or sheets of graphene extending outwards (Gao et at., 2011; Sreeprasad 2011). These dimensions of layers were found to be in the range of nano meters (nm), shown in Fig 7 (A) and (B) and the presence of wide pores in the surface are seen. This may be the reason for the good adsorption properties of GSC.

\section{Conclusion}

Graphene sand composite prepared through biosynthesized technique by means of anchoring of sugar particles upon the sand with no binder, the different characterizations techniques used for the identification

Fig. 6. (A) visible UV absorption spectrum of mud water. (B)Visible UV absorption spectrum of filtered mud water formation of graphene sand composite are SEM imaging, XRD analysis and FTIR spectroscopy and the adsorption test. The graphene sand composite and the classical 
results of this work are comparable and up to the standards, however further work can be performed as well.

\section{Acknowledgement}

Authors are great fully acknowledge Department of Materials Engineering and its lab facilities as well as research staff of NED University of Engineering and Technology for their support and help to complete this research work.

Conflict of Interest. The authors declare no conflict of interest

\section{References}

Abdelmajid Timoumi., Saleh Noaiman Alamri, Hatem Alamri. 2018. The development of $\mathrm{TiO}_{2}$ graphenesand nano-composite thin films for solar cells. Results in Physics, 11: 46-51.

Bykkam, S., Rao, V.K .,Thunugunta, T. 2013. Synthesis and characterization of graphene oxide and its antimicrobial activity against Klebseilla and Staphylococcus. Advanced Biotechnology and Research, 4: 216-225.

Chandra, J. P. 2010.Water-dispersible magnetite reduced graphene oxide composites for arsenic removal. ACS Nano 4: 3979-3986.

Chao, Xu. 2013. Graphene oxide- $\mathrm{TiO}_{2}$ composite filtration membranes and their potential application for water purification. Carbon, 62: 465-471

Chongyang, Qi., Lianqin, Z., Y, Lin., Deyi, W. 2018. Graphene oxide/chitosan sponge as a novel filtering material for the removal of dye from water Colloid and Interface Science, 517: 18-27

Dreyer, R., Sungjin, P. 2010. The chemistry of grapheme oxide. Chemical Society Reviews, 39: 228-240.

Dreyer, R., Sungjin, P., Christopher. 2012. The chemistry of graphene oxide. Chemical Society Reviews 39: 228-240.

Das. 2014. Carbon nanotube membranes for water purification: A bright future in water des-alination. Desalination, 336: 97-109.

Gao, W., Maju, M., der, Alemany, B. L. 2011. Engineered graphite oxide materials for application in water purification. ACS Appl. Material Interfaces, 3: 1821-1826.

Jisoo, P., Young., Sheik, C. 2018. Characteristics tuning of graphene-oxide-based-graphene to various enduses. Energy Storage Materials, 14: 8-21.

Lujaniene, G., Semcuk, S., Lecinsikyte, A., Kulakauskaite, I. 2017. Magnetic graphene oxide based nano-composites for removal of radio nuclides and metals from contaminated solutions. Environmental Radioactivity, 166: 166-174.

Peng, Li., Zhang, Bo., Tianhong, Cui. 2015. $\mathrm{TiO}_{2}$ and shrink induced tunable nano self-assembled graphene composites for label free biosensors. Sensors and Actuators B: Chemical, 216: 337-342.

Poornima Parvathi, V., Umadevi, M. 2016. Improved waste water treatment by bio-synthesized graphene sand composite. Environmental Management, 162: 299-305.

Renu, D.J., Bajpai., B. 2015. Green synthesis of graphene sand composite (GSC) as novel adsorbent for efficient removal of $\mathrm{Cr}(\mathrm{VI})$ ions from aqueous solution. Water Process Engineering, 5: 83-94.

Savage, N., Diallo, M.S. 2005. Nanomaterial and water purification: opportunities and challenges. Nanoparticle Research, 7: 331-342.

Sreeprasad, T.S. 2011. Reduced graphene oxide-metal/, metal oxide composites: facile synthesis and application in water purification. Hazardous Materials, 186: 921-931.

Song, N., Gao, X., Ma. Z., Wang, X. 2018. A review of graphene-based separation membrane: materials, characteristics, preparation and applications. Desalination, 437: 59-72.

Theruvak kattil Sreenivasan. 2013. Immobilized graphene-based composite from asphalt: facile synthesis and application in water purification. Hazardous Materials, 246- 247: 213-220.

Xiao, T., Yun-guo, L., Yan-ling, Gu., Yan, X. 2016. Biochar-based nano-composites for the decontamination of wastewater. Bioresource Technology, 212: 318-333.

Yang, S.T., Chang, Y. 2010. Aggregation of graphene oxide and its application in $\mathrm{Cu}^{2+}$ removal. Colloid and Interface Science, 351:122-127. 